\title{
Mejora docente en dolor agudo y crónico para el Grado de Odontología
}

\section{Teaching improvement in acute and chronic pain for the degree of dentistry}

JOSE MANUEL LóPEZ MILLÁN

ORCID: https://orcid.org/0000-0001-7999-2257

Universidad de Sevilla

Departamento de Cirugía

joselopezmillan@us.es

Fecha de recepción: 19/11/2019

Fecha de aceptación: 25/11/2019

DOI: http://dx.doi.org/10.12795/9788447221912.102

Pp.: 2287-2310 


\section{Resumen}

Ciclo de mejora docente en la asignatura de Patología Quirúrgica que se imparte en segundo curso de Grado de Odontología, aplicado al estudio y tratamiento del dolor, durante el primer cuatrimestre. Desde una reflexión sobre el modelo docente tradicional que utiliza el método transmisivo, el objetivo de la intervención es establecer un modelo más participativo para el alumno, donde el aula sea en un espacio para la discusión, el análisis de contenidos y la aplicación práctica de los mismos sobre situaciones clínicas concretas. El profesor adopta un papel de coordinación, facilitando las condiciones para que tenga lugar el aprendizaje orientado a la práctica profesional y centrado en el paciente. El rediseño del modelo tradicional parte de la formulación de preguntas clave que definan los aprendizajes deseables e incluye la revisión de contenidos, el diseño de actividades de contraste y una evaluación de la progresión del aprendizaje.

Palabras clave: patología quirúrgica, odontología, docencia universitaria, experimentación docente universitaria, dolor.

\section{Abstract}

Teaching improvement cycle in the subject of Surgical Pathology taught in the second year of the Degree in Dentistry, applied to the study and treatment of pain, during the first semester. From a reflection on the traditional teaching model that uses the transmissive method, the objective of the intervention is to establish a more participatory model for the student, where the classroom is a space for discussion, content analysis and their practical application on specific clinical situations. The teacher adopts a coordinating role, facilitating the conditions to the for professional-oriented and patient-centered learning to take place. The redesign of the traditional model is based on the formulation of key questions that define desirable learning and includes the review of content, the design of contrast activities and an evaluation of the progression of learning.

Key words: surgical pathology, dentistry, university teaching, university teaching experimentation, pain. 


\section{Descripción del contexto}

El ciclo de mejora docente se aplica al Área de Anestesiología-Reanimación y Tratamiento del Dolor de la asignatura Patología Quirúrgica, que se imparte en el segundo curso del Grado de Odontología. La asignatura tiene carácter obligatorio y se desarrolla en el primer cuatrimestre. Es de tipo teórico-práctico aunque la práctica se realiza en aula en forma de seminario, separada de las clases teóricas en una relación 1:5 en cuanto al tiempo dedicado. Los alumnos de segundo curso se organizan en dos grupos de 39 y 44 alumnos respectivamente tanto para la teoría como para las prácticas. Las clases se desarrollan en un aula con distribución en bloque y tienen lugar los martes a las 8:00 para el grupo A y a las 13:00 para el grupo B. Este ciclo de mejora docente se aplica a un bloque de contenido que incluye la fisiopatología, valoración y el tratamiento del dolor agudo y crónico. Este bloque se imparte en cuatro horas lectivas de contenido teórico entre los meses de octubre y noviembre de 2019.

\section{Diseño previo del Ciclo de Mejora Docente}

\section{Mapa de contenidos y problemas}

El mapa de contenidos tiene como finalidad ayudar al alumno a dar respuesta a preguntas fundamentales que constituyen el eje central del bloque de materia que estamos abordando en este segundo CIMA. Estas preguntas son: 1. Qué es el dolor, 2. Diferencia entre sensación, emoción y experiencia, 3. ¿Podemos tener dolor estando anestesiados localmente?, 4. Cómo afecta el dolor a nuestro estado de ánimo y viceversa, 5. Cómo es el proceso de experimentar dolor, 6. Cómo puedo afrontar y orientar a un paciente con dolor, 7. Cómo usar una escala o un 
cuestionario de dolor, 8. Cómo hacer un diagnóstico de dolor, 9. Cómo puedo establecer un tratamiento analgésico, 10. Identificar, manejar y derivar al paciente con una neuralgia del nervio trigémino.

El mapa de contenidos hace referencia a la parte de la asignatura que es objeto del segundo ciclo de mejora docente, esto es, la fisiopatología, valoración y tratamiento del dolor agudo y crónico.

Listado de contenidos agrupados por categorías:

a) Conceptos: qué es el dolor, nocicepción, sufrimiento, sensación y emoción, experiencia, agudo y crónico, nociceptivo y neuropático, modelo biopsicosocial, afrontamiento y adaptación, neuromodulación.

b) Datos: clasificación del dolor, receptores, fibras, láminas, vías espinales, matriz cerebral, etapas del proceso nociceptivo, neurotransmisores, descriptores de dolor neuropático, grupos de fármacos y técnicas de analgesia.

c) Procedimientos/habilidades: anamnesis dirigida, exploración neurológica, uso de cuestionarios y escalas, diagnóstico, información y toma de decisiones compartida, técnicas, tratamiento y recomendaciones en salud.

d) Valores/actitudes: misión y visión estratégica, empatía, escucha activa, comunicación, respeto por la autonomía del paciente, conducta, juicios, servicio social, educación para la salud.

Las relaciones entre los diferentes contenidos quedan representadas en el mapa mediante colores y flechas. Los colores representan las diferentes categorías de los contenidos y las flechas la relación que existe entre ellos. Se ha sombreado agrupaciones de contenidos que tienen otro nivel de relación en base a la sesión docente en la que fueron abordados y tienen un orden cronológico de izquierda a derecha en el mapa. Los contenidos han sido 
distribuidos siguiendo un orden jerárquico decreciente de arriba a abajo y de izquierda a derecha de una forma general. Se ha intentado reducir contenido al máximo para adaptarlo a la nueva estructura de las sesiones docentes de forma que se conceda más tiempo al alumno y su interacción con los contenidos y con las opiniones de otros alumnos a través de actividades de contraste. Los números 1 a 10 del mapa se refieren a las preguntas a las que los contenidos pretenden dar respuesta.

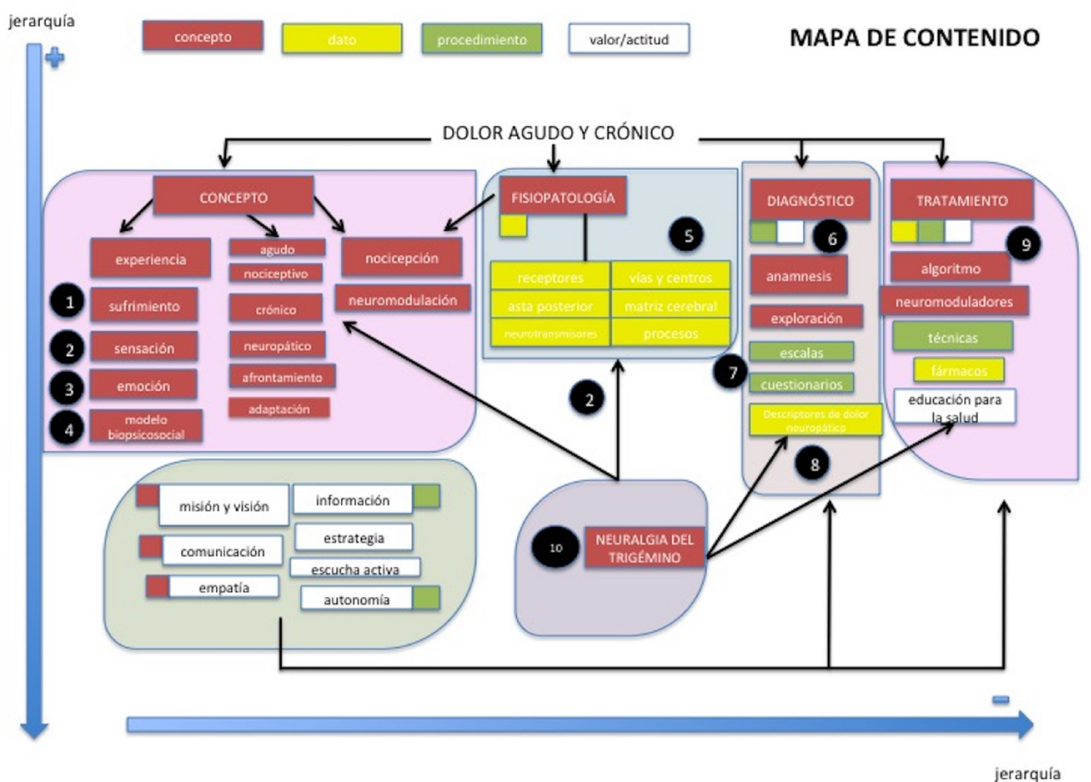

Figura 1. Mapa de Contenidos

\section{Modelo metodológico posible}

Para la elaboración del modelo metodológico posible he realizado una reflexión crítica sobre el modelo didáctico actual siguiendo las recomendaciones de García Pérez y Porlán en el capítulo 5: Los principios didácticos y el modelo didáctico personal del libro "Enseñanza Universitaria. Cómo mejorarla" (Ed. Morata, 2017). En dicho análisis contemplo desde la perspectiva del alumno y su aprendizaje, los distintos aspectos didácticos, psicológicos y de 
conocimiento que constituyen la acción docente en mi práctica habitual para intentar darle una vuelta en 360 en la medida de lo posible.

Fruto de dicho análisis elaboro un modelo metodológico posible, representado en la Figura 2, en el cual, las situaciones problemáticas (SP) se plantean al alumno mediante un caso clínico real escrito, o una encuesta personal sobre sus experiencias y creencias previas sobre el tema. Ambos ejercicios hará al alumno pensar para resolver individualmente, antes de abordar el tema teórico, utilizando los conocimientos preclínicos adquiridos en el curso anterior y una elevada dosis de sentido común. Para el docente, este ejercicio permitirá acercarse al punto de partida del estudiante y establecer una estrategia de acercamiento e inmersión en el proceso de aprendizaje.

Los modelos iniciales del alumno (E1) se expondrán individualmente elegidos al azar entre los más representativos.

A continuación (T/P) los modelos serán discutidos por el grupo de forma ordenada, con la moderación del profesor que irá introduciendo conceptos teóricos orientativos al tiempo que cuestiones prácticas que permitan el planteamiento de nuevas hipótesis y alternativas de discusión con una orientación práctica hacia la resolución del problema de salud planteado.

La nueva versión de modelos del alumno (E2) consistirá en la exposición a modo de resumen por parte de dos o tres alumnos de su versión final del caso de manera que quede una solución abierta al problema. En el caso que se haya realizado un cuestionario o encuesta sobre conocimiento o experiencias previas, al final de la clase se comentarán al azar algunos de los más representativos con la perspectiva adquirida durante la sesión. 


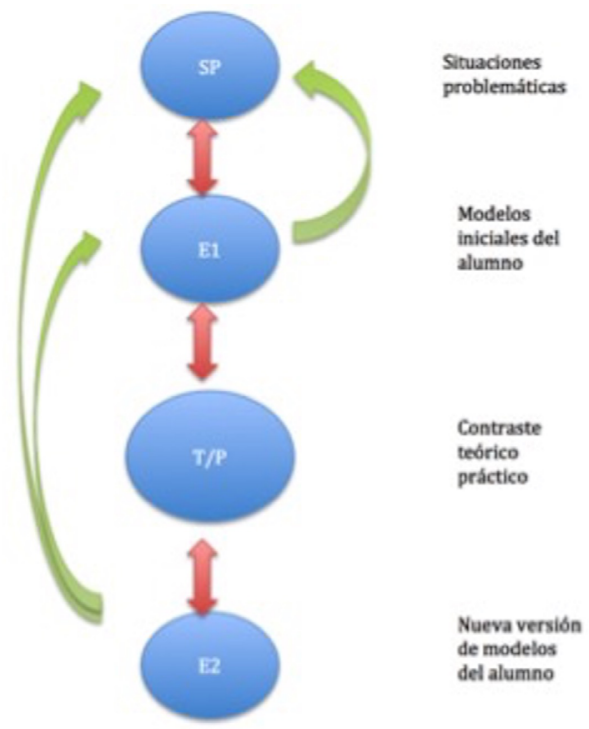

Figura 2. Representación del modelo metodológico posible.

En este modelo, el preámbulo se realiza presentando el caso clínico o el cuestionario y planteando sus objetivos. Se cede más tiempo al alumno tanto individual como colectivamente en un entorno más participativo invitándole a presentar la resolución de su caso. Tanto la asistencia a clase como la participación son vinculantes para la nota final además del examen teórico.

El contenido teórico, fundamentalmente conceptual y actitudinal se va introduciendo a lo largo de la discusión del caso para completar su resolución colectiva al tiempo que se abordan los aspectos fundamentales del tema.

El cierre del tema lo hacen los alumnos a través de una exposición de los modelos más representativos seleccionados por el profesor durante la clase.

Se suprimen las lecturas para hacer en casa y solo se entrega la presentación tipo elaborada por el profesor, que se ha utilizado en clase en Power Point, con el contenido total del tema. En ésta se incluyen las referencias 
bibliográficas para que el estudiante pueda ampliar contenidos si lo desea.

Tabla 1. Secuencia de actividades en cada sesión

\begin{tabular}{|c|c|c|c|}
\hline $\begin{array}{l}\text { Problema } \\
\text { Subproblema } \\
\text { Sesión }\end{array}$ & $\begin{array}{l}\text { Tipo } \\
\text { de } \\
\text { Sesión }\end{array}$ & Descripción & $\begin{array}{c}\text { Duración } \\
\text { (min.) }\end{array}$ \\
\hline \multirow{4}{*}{$\begin{array}{l}\text { 1. Dolor Agudo y Crónico } \\
\text { 1.1 Fisiopatología de la } \\
\text { nocicepción: } \\
\text { · } \text { Qué es el dolor } \\
\text { - } \quad \text { Diferencia entre sensación, } \\
\text { emoción y experiencia } \\
\text { - ¿Podemos tener do- } \\
\text { lor estando anestesiados } \\
\text { localmente? } \\
\text { - Cómo afecta el dolor a } \\
\text { nuestro estado de ánimo y } \\
\text { viceversa } \\
\text { - Cómo es el proceso de ex- } \\
\text { perimentar dolor } \\
\text { Sesión 1: anatomía funcional, } \\
\text { teoría de la puerta de entrada, } \\
\text { modelo biopsicosocial }\end{array}$} & $\begin{array}{l}\text { Situación } \\
\text { Problema: } \\
\text { evaluación } \\
\text { inicial }\end{array}$ & $\begin{array}{l}\text { Se presenta un } \\
\text { cuestionario con } 5 \\
\text { preguntas sobre dolor. }\end{array}$ & 5 \\
\hline & $\begin{array}{l}\text { Resolución } \\
\text { Individual }\end{array}$ & $\begin{array}{l}\text { Se pide al alumno que } \\
\text { la analice, y comente } \\
\text { alguna experiencia que } \\
\text { llame su atención. }\end{array}$ & 15 \\
\hline & $\begin{array}{l}\text { Discusión } \\
\text { Teórico- } \\
\text { práctica }\end{array}$ & $\begin{array}{l}\text { Introducción por el } \\
\text { profesor de elementos } \\
\text { conceptuales que } \\
\text { pudieran explicar } \\
\text { dichas experiencias } \\
\text { para conectar creencias } \\
\text { con ciencia y cómo } \\
\text { los investigadores } \\
\text { pensando lo que ellos } \\
\text { piensan pudieron } \\
\text { llegar a lo que hoy de } \\
\text { momento conocemos } \\
\text { en base al método } \\
\text { científico. }\end{array}$ & 20 \\
\hline & $\begin{array}{l}\text { Nueva } \\
\text { versión de la } \\
\text { Resolución }\end{array}$ & $\begin{array}{l}\text { Comentario a modo } \\
\text { de cierre de algunas } \\
\text { experiencias más } \\
\text { representativas. }\end{array}$ & 10 \\
\hline $\begin{array}{l}\text { 1. Dolor Agudo y Crónico } \\
\text { 1.2 Clínica: } \\
\text { Cómo puedo afrontar y } \\
\text { orientar a un paciente con } \\
\text { dolor } \\
\text { - Cómo usar una escala o un } \\
\text { cuestionario de dolor } \\
\text { Sesión 2: Signos y síntomas }\end{array}$ & $\begin{array}{l}\text { Situación } \\
\text { Problema }\end{array}$ & $\begin{array}{l}\text { Se presenta un caso } \\
\text { clínico (1) de dolor } \\
\text { agudo postoperatorio } \\
\text { tras cirugía oral. }\end{array}$ & 5 \\
\hline
\end{tabular}

Jornadas de Formación e Innovación Docente del Profesorado I № 2 (2019) Esta obra se distribuye con la licencia Creative Commons Reconocimiento-NoComercial-SinObraDerivada Internacional (CC BY-NC-ND 4.0.) 


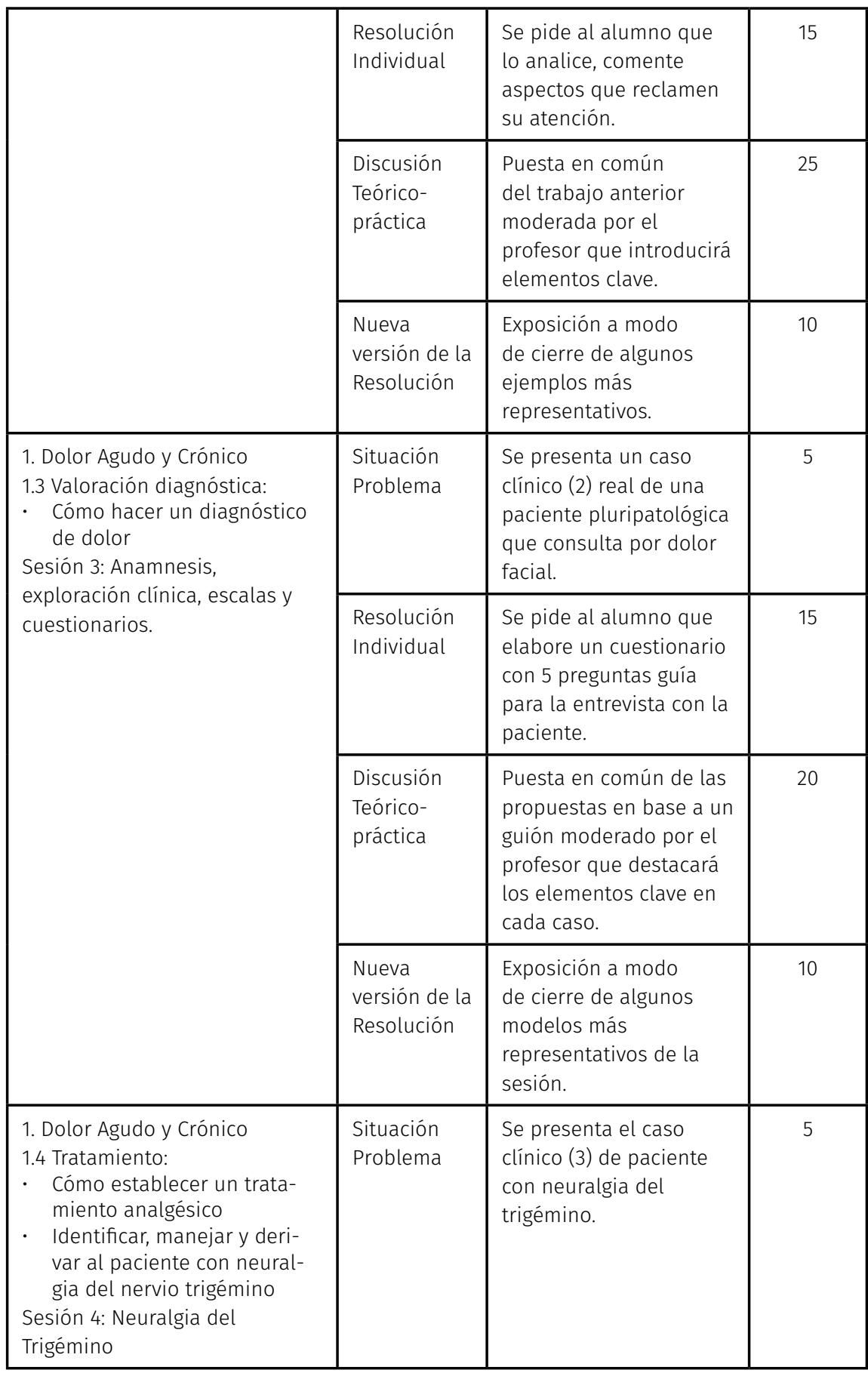




\begin{tabular}{|l|l|l|c|}
\hline & $\begin{array}{l}\text { Resolución } \\
\text { Individual }\end{array}$ & $\begin{array}{l}\text { Se pide al alumno } \\
\text { analice, realice una } \\
\text { hipótesis diagnóstica } \\
\text { y un tratamiento } \\
\text { potencial. }\end{array}$ & 15 \\
\cline { 2 - 5 } & $\begin{array}{l}\text { Discusión } \\
\text { Teórico- } \\
\text { práctica }\end{array}$ & $\begin{array}{l}\text { Puesta en común de } \\
\text { las propuestas en base } \\
\text { a un guión moderado } \\
\text { por el profesor que } \\
\text { recogerá los principales } \\
\text { aspectos con énfasis en } \\
\text { su tratamiento. }\end{array}$ & 20 \\
\cline { 2 - 6 } & $\begin{array}{l}\text { Nueva } \\
\text { versión de la } \\
\text { Resolución }\end{array}$ & $\begin{array}{l}\text { Exposición a modo } \\
\text { de cierre de algunos } \\
\text { modelos más } \\
\text { representativos de la } \\
\text { sesión. }\end{array}$ & 5 \\
\hline & $\begin{array}{l}\text { Se presenta un } \\
\text { cuestionario con 5 } \\
\text { preguntas sobre dolor } \\
\text { que se presentó al } \\
\text { comienzo de la primera } \\
\text { sesión }\end{array}$ & 5 \\
\hline $\begin{array}{l}\text { Situación } \\
\text { problemaación } \\
\text { final }\end{array}$ & 5
\end{tabular}

\section{El caso clínico 2 se puede tomar como ejemplo de caso} tipo utilizado en las sesiones:

“Paciente de sexo femenino, de 73 años de edad, no hábitos, con antecedentes personales de hipertensión arterial bien controlada, dislipemia en tratamiento, bronquitis crónica de origen asmático sin crisis desde hace tres meses, diabetes mellitus en tratamiento con antidiabéticos orales, con obesidad moderada y sindrome de apnea obstructiva del sueño que consulta por dolor facial de tres meses de evolución en la región mandibular derecha de características punzantes, como una frialdad interior que le recorre la cara desde el mentón hasta el oído derecho, a veces en forma de descargas que alterna con períodos de ausencia de dolor sin patrón definido aunque reconoce que empeora al masticar por ese lado y cuando se roza la cara al lavarse o al maquillarse. No ha tenido fiebre y no 
presenta ninguna otra sintomatología aparentemente que el paciente refiera. Interfiere con el sueño pues la despierta por la noche en ocasiones.

El paciente dice tener dolor desde hace muchos meses pero lo atribuía a piezas dentales en mal estado que han ido siendo reparadas o extraídas en este tiempo, pero después de finalizar los procedimientos el dolor persiste incluso más intenso y desagradable.

Ha tomado analgésicos menores y su médico de cabecera le ha prescrito tramadol pero no encuentra alivio significativo.

El examen oral es normal aparentemente y la ortopantomografía no muestra hallazgos de interés salvo varias exodoncias, una de ellas coincidente con la zona mandibular derecha."

\section{Cuestionario inicial-final para hacer seguimiento de los estudiante}

Al principio y al final del ciclo de mejora docente se propuso a los alumnos completar el siguiente cuestionario breve con carácter anónimo, compuesto por cinco preguntas cortas sobre el dolor:

"Por favor contesta brevemente a las siguientes preguntas sobre el dolor.

1. En tu opinión: ¿Qué es el dolor?

2. Explica la diferencia entre un dolor agudo y otro crónico.

3. Piensa en el peor dolor que hayas experimentado en tu vida y describe sus características y cómo te sentías en ese momento.

4. ¿Qué preguntarías a un paciente que acude a ti por dolor?

5. ¿Se puede tener dolor en una zona del cuerpo carente de sensibilidad?" 


\section{Aplicación del Ciclo de Mejora Docente}

\section{Relato resumido de las sesiones}

El relato toma como base el conjunto de anotaciones realizadas durante y después de cada sesión a modo de diario del profesor.

- Primera sesión: la asistencia a clase es del 60\% a primera hora, en el grupo A y del $87 \%$ en el grupo B a última hora de la mañana. Comienza la sesión con un cuestionario sobre dolor agudo y crónico que incluye preguntas conceptuales y una pregunta autorreferencial con el objetivo de hacer reflexionar al alumno sobre el tema, despertar su interés y permitirme conocer sus esquemas mentales sobre el mismo. Los alumnos se mostraron motivados con la tarea que realizaron adecuadamente. En la puesta en común hay resistencia a la participación, que requiere que tenga que ir preguntando a cada uno por aspectos concretos del cuestionario. Siempre participan los mismos y decido hacer un muestreo aleatorio que por falta de tiempo y al no conocer el contenido de las respuestas, no es representativo, sin embargo permite que haya discusión y mejore la participación. Llama la atención lo dificil que resulta hablar sobre el dolor a pesar de que todos lo han experimentado alguna vez en su vida. En general hablar sobre emociones es complicado frente a hablar de experiencias, tendiendo en este último caso a abordar las experiencias desde el hacer más que desde el sentir. En muchos casos los alumnos no tienen clara la diferencia entre sensibilidad y dolor entendiendo que cuando se altera una, se afecta la otra. 0 la diferencia entre nivel de conciencia, mientras dormimos por ejemplo, y la capacidad para experimentar dolor. La cuestión que plantea más dificultad es 
la de experimentar dolor sobre una zona del cuerpo carente de sensibilidad. Durante la puesta en común de las propuestas voy interviniendo para introducir conceptos como emoción y sensación, experiencia, memoria y aprendizaje, biografía y umbral sensitivo, especialización sensorial, neuromodulación espinal y cerebral, teoría de la puerta de control, matriz cerebral, sufrimiento, empatía y simpatía, comunicación y vertiente social del dolor. En el comentario a modo de cierre se generan tres intervenciones intentando contrastar ideas utilizando los conceptos expuestos durante la clase de una manera muy coherente. La sesión es densa conceptualmente e intensa en cuanto al ritmo de la misma durante los 55 minutos.

- Segunda sesión: asistencia a clase es del $62.7 \%$ a primera hora, en el grupo A y del $82.05 \%$ en el grupo B a última hora de la mañana. Cuatro alumnos exponen su visión durante la sesión, todos ellos autorreferenciales. Apoyándome en los conceptos expuestos en la primera sesión, voy enlazando aspectos particulares del caso con dichos conceptos para ir añadiendo complejidad progresiva al caso con el objetivo de mostrar que en dolor casi nunca es lo que parece porque la presentación de los síntomas es muy variable entre pacientes aun en un dolor postoperatorio que es el ejemplo de dolor estándar. Para mi satisfacción, observo que los estudiantes apenas toman apuntes, algo que no había advertido en la sesión anterior pero que me llamó poderosamente la atención en el primer el anterior ciclo de mejora pues es la tónica en estos cursos de grado. Detecto un clima de confianza y en general de relajación en las caras de algunos alumnos cuando me dirijo a ellos. Se trata de una sesión conceptual que nos lleva de la fisiología a la fisiopatología de la nocicepción, los tipos de dolor, las diferentes clasificaciones conceptuales y los patrones más habituales 
de presentación. Llama la atención a los alumnos la terminología utilizada para describir alteraciones de la sensibilidad como alodinia, hiperalgesia, hipoestesia, desconocida para la mayoría, lo cual me hace volver a la idea de que no estoy ante médicos, ni ante alumnos de cuarto curso de grado de medicina (el otro grupo al que doy clase) sino con estudiantes de segundo curso de odontología, en una asignatura preclínica o de iniciación a la clínica. Concluimos la sesión destacando la importancia de observar y escuchar al paciente de una manera abierta para captar todos los matices que este presente clínicamente prestando atención a aspectos sutiles que pueden marcar la diferencia en el diagnóstico.

- Tercera sesión: la asistencia a clase es del $55.8 \%$ a primera hora, en el grupo A y del $94.87 \%$ en el grupo $\mathrm{B}$ a última hora de la mañana. No pueden entrar en clase 5 alumnos del grupo A por llegar comenzada la clase. Esta sesión pretende presentar casos reales habituales y la relación que existe entre el estado fisico del paciente, el dolor que presenta y cómo todo ello condiciona la actuación profesional del odontólogo tanto en el diagnóstico como en el tratamiento. Varios alumnos exponen sus preguntas dirigidas sobre todo a identificar la intensidad del dolor y su localización. Solo en un caso se pregunta por situaciones o acciones que lo empeoran o mejorar. Solo un alumno pregunta por el tiempo de evolución y otro pregunta por la causa o posibles causas. $\mathrm{Na}$ die pregunta por las características del dolor o la sintomatología asociada. La idea central es que el diagnóstico del dolor es clínico y se basa en una entrevista dirigida y en la exploración del paciente que incluye tanto aspectos colaterales al motivo de consulta como aspectos biográficos, es decir, antecedentes personales de interés. Continuamos con la exploración clínica sensorial y la diferencia del dolor espontáneo y evocado. Destaco la importancia de 
tocar al paciente. Todo ello mejora nuestra compresión y a su vez nuestra relación con el paciente. Presento dos ejemplos de escalas y cuestionarios que comentamos en grupo y discutimos su aplicación y utilidad clínica. El cuestionario DN 4 lo presento en inglés, lo cual tengo la impresión de que es una limitación pues se centran más en traducirlo que en lo que representa y valora.

- Cuarta sesión: la asistencia a clase es del $69.9 \%$ a primera hora, en el grupo A y del $92.15 \%$ en el grupo B a última hora de la mañana. Se cumple la puntualidad al inicio y final de la sesión. A través de la participación de algunos alumnos entiendo que el nivel de compresión del caso es adecuado y presenta gran curiosidad, incluso diría que provoca cierto respeto, sobre todo a la hora de proponer acciones concretas a llevar a cabo que no es obviamente el objetivo final de la sesión. En la discusión del caso puedo completar aspectos de sesiones anteriores relacionados con la sintomatología, el diagnóstico y los procesos neurobiológicos que subyacen a las manifestaciones del paciente, así como las múltiples causas que pueden desencadenar o contribuir a la experiencia de dolor crónico. Pongo de relieve la importancia de "no hacer" en algunas ocasiones frente a "hacer" algo que puede ser perjudicial para el paciente ("hacer daño") cuando no se tiene clara la situación clínica. Estamos predispuestos para la acción y nuestro ego profesional nos lleva a ello siendo más dificil encontrar razones para no actuar en determinadas situaciones. Se pone de relieve en esta sesión la importancia de las comorbilidades del paciente y su papel en la evolución y el pronóstico de la patología que motiva la consulta. Las conclusiones de la sesión van dirigidas a cómo establecer un diagnóstico sindrómico de sospecha y un plan general de tratamiento que incluya informar y tranquilizar al paciente, hacer educación para la salud, iniciar un tratamiento farmacológico general y derivar para estudio. 


\section{Evaluación del aprendizaje de los estudiantes}

El cuestionario breve anónimo fue contestado por los estudiantes al principio y al final de módulo de clases con un porcentaje representativo del total de alumnos del curso, 70,2\% y 66,6\% respectivamente. En base al cuestionario breve de cinco preguntas cortas se elabora una escalera de cinco niveles de dificultad para agrupar las respuestas proporcionadas por los estudiantes en cada pregunta. Se han descartado aquellas respuestas poco representativas por su contenido o por su frecuencia de aparición y se ha establecido que cada nivel tiene proporcionalmente el mismo nivel de dificultad respecto al anterior, siendo éste progresivamente creciente desde el nivel 1 al cinco. Dicho nivel de dificultad viene representado por la altura del peldaño de la escalera. El ancho de cada peldaño lo determina el porcentaje estudiantes cuyas respuestas se identifican con cada nivel de conocimiento y es lo que representa el porcentaje calculado sobre el número de respuestas similares en cada nivel de conocimiento.

Veámos como ejemplo el análisis de la pregunta 1 del cuestionario "En tu opinión, ¿qué es el dolor?". Las respuestas de los estudiantes se han agrupado en cinco categorías de menor a mayor complejidad como se muestran las figuras 3 y 4 . La figura 3 representa la escalera elaborada a partir de las respuestas del cuestionario inicial donde la mayoría de los estudiantes incluyen en sus respuestas aspectos más básicos del dolor y relacionados con conceptos sensoriales de la percepción siendo menos frecuentes las respuestas que aluden a aspectos emocionales, subjetivos, individuales, de limitación funcional y pérdida de la calidad de vida.

Dado que las respuestas abiertas de cada estudiante incluye varias ideas, el porcentaje representa el número de respuestas relacionadas con cada nivel de conocimiento observando cuántos alumnos responden de 
acuerdo con cada nivel de complejidad para esta pregunta. En la pregunta primera una mayoría de alumnos responde adecuadamente en el nivel básico, mientras solo dos alumnos lo hacen con el máximo nivel de complejidad.

ESCALERA CONOCIMIENTO. PREGUNTA 1 inicial.

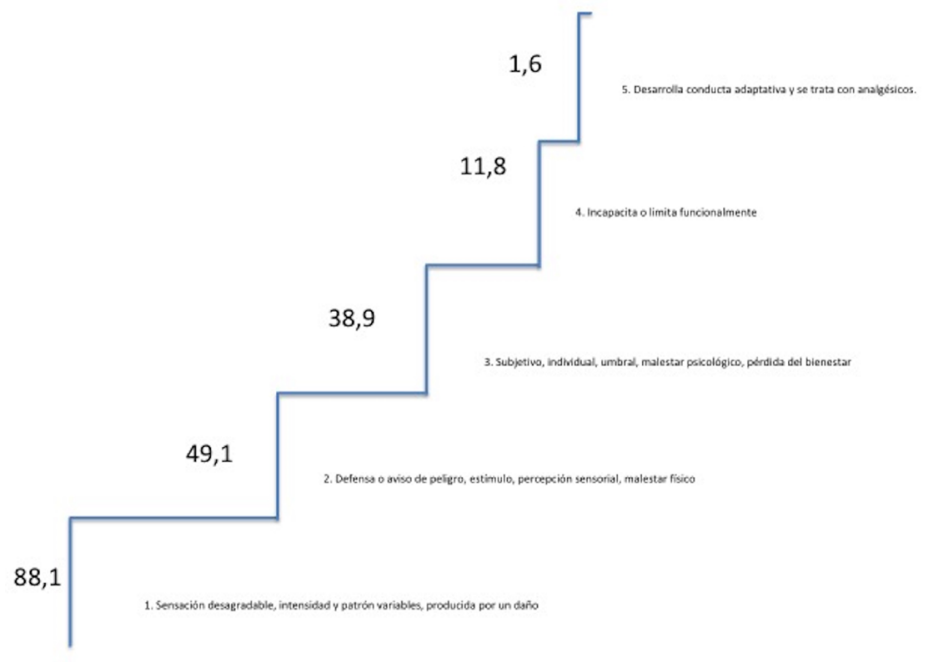

Figura 3. Escalera de aprendizaje de la pregunta 1 al inicio del Ciclo de Mejora.

La figura 4 representa la escalera elaborada a partir de las respuestas del cuestionario final. En ella podemos ver que el porcentaje de alumnos que responde al primer y segundo nivel es algo inferior al cuestionario inicial pero la tendencia se invierte para los niveles tercero y cuarto con mayor nivel de dificultad quedando prácticamente igual en el quinto nivel donde solo un alumno en cada grupo alude a cuestiones relacionadas con el tratamiento farmacológico y aspectos conductuales desarrollados por los pacientes con dolor.

Las respuestas de los niveles tercero y cuarto son más directas y tienen una base en el dolor agudo y en la experiencia cotidiana de personas jóvenes con apenas 
experiencias de dolor crónico severo por lo que se encuadran más en esta perspectiva. El hecho de que el número de respuestas en estos niveles se vea ligeramente reducido en el cuestionario final puede estar relacionado con el abordaje del contenido desde la perspectiva del dolor crónico que profundiza más en aspectos emocionales de la experiencia de dolor pudiendo justificar este hecho el aumento de las respuestas en los niveles tercero y cuarto con mayor nivel de dificultad pues sitúa al estudiante ante una vertiente más compleja del dolor conectándolo con las emociones, la pérdida del bienestar y de la calidad de vida.

El quinto nivel de complejidad no incluye apenas una respuesta en cada grupo pues si bien se han abordado estos contenidos en clase, se han considerado de un carácter jerárquico inferior por tratarse de una asignatura preclínica siendo estos aspectos abordados en cursos posteriores del grado.

ESCALERA CONOCIMIENTO. PREGUNTA 1 final.

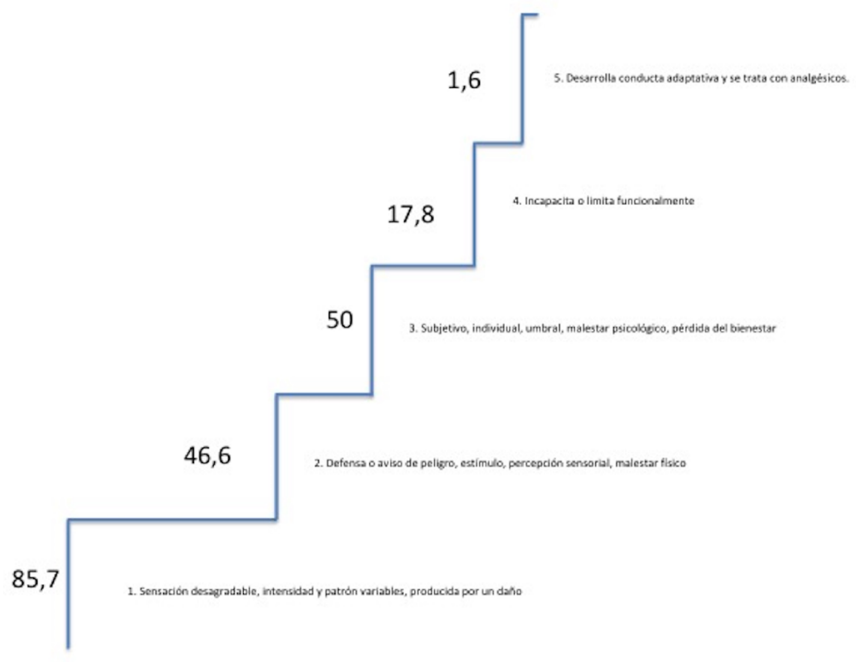

Figura 4. Escalera de aprendizaje de la pregunta 1 al final del Ciclo de Mejora.

Jornadas de Formación e Innovación Docente del Profesorado | № 2 (2019) Esta obra se distribuye con la licencia Creative Commons 
Debido al número de estudiantes y al carácter anónimo del cuestionario, no se ha elaborado un cuadro de evolución por estudiante sino que se ha realizado un análisis del conocimiento colectivo sobre el tema. En líneas generales podemos decir que el cuestionario no discrimina adecuadamente el nivel de conocimiento previo y posterior del alumno en parte por el número reducido de preguntas. Sin embargo, se observa una evolución en el grado de conocimiento de los estudiantes que se sitúan en niveles de complejidad superiores en todas las preguntas del cuestionario. Este hecho va en consonancia con el diseño de actividades orientadas a trabajar aspectos conceptuales complejos frente a los esquemas cotidianos arquetípicos sobre el dolor que tienen los estudiantes al inicio del ciclo.

\section{Evaluación del CMD puesto en práctica:}

\section{Evaluación de la docencia por los estudiantes.}

Para conocer la opinión de los alumnos sobre la metodología docente, he preferido centrarme en el segundo CIMA por tenerlo más reciente y diferir en algunos aspectos del primero.

He utilizado una encuesta simple con una sola pregunta de tres opciones para elegir la que más se ajuste a su opinión: a) Me ha gustado, b) No me ha gustado, c) Y si... (propuesta)

El sondeo a los alumnos se realizó a través de la plataforma de enseñanza virtual y ha tenido un seguimiento significativo con una participación del $73.4 \%$.

El resultado de la encuesta es el siguiente: 
- al $79,1 \%$ de los alumnos le ha gustado el planteamiento de la asignatura en este bloque de contenido.

- al 7,2\% de los alumnos no ha gustado el planteamiento de la asignatura en este bloque de contenido.

- un $13,6 \%$ de los alumnos propondrían cambios en su mayoría orientados a aumentar el contenido práctico de la asignatura y reducir el contenido teórico y la densidad de las sesiones.

\section{Cuestiones a mantener y cambios a introducir para un futuro Ciclo de Mejora}

De forma general considero que necesito aumentar la duración de las sesiones para abordar los aspectos conceptuales combinando los casos, el cuestionario y el contraste con trabajo en grupo o por parejas utilizando la técnica del juego de rol que es muy útil para recrear situaciones clínicas reales. La organización y distribución del aula tampoco lo permite.

Son mejorables algunas diapositivas, reducir otras que no aportan información relevante y en particular no utilizar ejemplos de cuestionarios en inglés.

Creo que es necesario rediseñar el mapa de contenidos para pulirlo un poco más de manera que quede más redondo el abordaje del bloque de materia lo cual, a su vez, me permitirá ir más al grano y ajustarme más al tiempo. He reducido mucho contenido teórico y memorístico pero es cierto que este tipo de contenido consume poco tiempo mientras que al aumentar contenido conceptual de mayor jerarquía y actividades de contraste, se incrementa la necesidad de tiempo siendo esta, en mi opinión, la razón por la que aumenta la densidad de las sesiones y se necesita más tiempo para mejorar el aprendizaje. 
Por otra parte, sería interesante poder incorporar una sesión a modo de presentación del bloque de contenido para indagar sobre los conocimientos que tiene el alumno al principio del curso, rediseñar el modelo metodológico y exponerlo antes de comenzar de manera que estén más familiarizados con el mismo.

\section{Aspectos de la experiencia que se pretenden incorporar a toda la práctica docente habitual}

El aspecto fundamental que me gustaría incorporar es la exploración de los modelos conceptuales del alumno y su motivación para el aprendizaje. En segundo lugar me gustaría mejorar el diseño de actividades que respondan más adecuadamente a los problemas clave que plantea la asignatura. Y por último, introducir un sistema de evaluación continua.

\section{Principios Didácticos argumentados que han guiado la experiencia y que deben permanecer en el futuro.}

Principios didácticos como base de mi modelo metodológico posible:

- Finalidad: sensibilizar al alumno con un problema y sus posibles soluciones, conectándolo con la realidad a través de un pensamiento crítico y abierto para la resolución de problemas que plantean los pacientes. Los modelos mentales individuales como punto de partida para reformularlos en base a unos contenidos y utilizando una metodología concreta para mejorar año tras año y reformular el modelo didáctico.

- Contenidos: trabajar aspectos conceptuales, metodológicos y actitudinales para que se sientan capaces de afrontar cualquier problema. Utilizar un mapa 
de contenidos hace que todo tenga más sentido y la información sea más coherente e integrada, facilitando el acceso a la misma al alumno. Creo que es un aspecto a mejorar de forma colectiva con mis compañeros de asignatura para el próximo cuatrimestre.

- Metodología: a partir de conceptos personales y creencias sociales, a través de sus propias experiencias, de las de otros compañeros y de casos clínicos, poder afrontar problemas más diversos y complejos. Este ha resultado el aspecto más complicado pues las actividades que he intentado desarrollar en clase no eran muy variadas y creo que han resultado poco productivas. Formular preguntas clave y elaborar una secuencia de actividades ad hoc tal vez sea la parte más dificil del programa y he echado en falta algunos ejemplos relacionados con mi asignatura. A pesar del trabajo realizado, no he conseguido que haya mucha participación en clase. La distribución del aula en bloque y la corta duración de las clases, 50 minutos, no permitieron mucho juego.

- Evaluación: evaluar el punto de partida como experiencia reflexiva, aunque calificando el proceso final de adquisición de nuevos esquemas conceptuales a través de un examen conceptual y actitudinal con la introducción de algún caso clínico real. He seguido un cuestionario breve anónimo que los estudiantes han contestado mayoritariamente. He cometido el error de no evaluar la primera ronda hasta haber realizado la segunda con lo cual no me he beneficiado de la información inicial para diseñar el mapa de contenido y las tareas que ya las tenía diseñadas al comenzar el segundo ciclo de mejora. Es importante realizar este tipo de cuestionarios antes de diseñar el ciclo y para poder aplicarlo utilizando alguna herramienta de sondeos que facilite la realización y el análisis posterior.

- Relaciones profesor-estudiante: equilibrada tanto en el trato personal como en el nivel de compromiso 
con el proceso docente pero centrada en el alumno en cuanto a que ellos establecen el punto de partida y el nivel de participación en el proceso.

- Aprendizaje: el docente adopta una posición de acompañamiento y adaptación de las herramientas y los contenidos al proceso de aprendizaje en función del punto de partida y su desarrollo. Ha de buscar la estrategia más adecuada para conseguir el mejor resultado posible a través de un proceso de mejora continua que tiene un objetivo abierto.

- Teoría sobre el conocimiento: se trata de deshacernos de posicionamientos dogmáticos pues aunque se trata de una ciencia con postulados más o menos estables avalados por el método científico, dichos postulados no son fijos sino que están sujetos a continua reevaluación y revisión; este espíritu crítico debe predominar sobre la asunción del dogma y debe guiar al alumno en todo el proceso de aprendizaje. Pero además, si pensamos en la vertiente humanística de las ciencias biosanitarias, es aún más importante si cabe conceder un carácter relativo a todo lo que se enseña si queremos encontrar nuevas perspectivas que no solo enriquezcan la experiencia sino que se adapten a las necesidades de la sociedad, al servicio de la cual está tanto el docente como lo estará el alumno. 


\section{Referencias bibliográficas}

Porlán, R. (Coord.) (2017). Enseñanza Universitaria. Cómo mejorarla. Madrid: Ediciones Morata S.L.

Finkel, D. (2008). Dar clase con la boca cerrada. Valencia: Publicacions de la Universitat de València.

Bain, K. (2007). Lo que hacen los mejores profesores universitarios. Valencia: Publicacions de la Universitat de València.

Porlán, R. (1993). Constructivismo y escuela. Hacia un modelo de enesñanza-aprendizaje basado en la investigación. Sevilla: Díada.

Jornadas de Formación e Innovación Docente del Profesorado | № 2 (2019) Esta obra se distribuye con la licencia Creative Commons 